\title{
An Advanced Replacement Model for a Two-Unit System
}

\author{
Xiaoyang Ma, Xiaodong Zhang, Rui Peng* \\ Donlinks School of Economics and Management, University of Science and Technology Beijing, China
}

\begin{abstract}
This paper considers an advanced replacement model for a two-unit system. One is the key unit called unit 1 and the whole system is replaced once unit 1 fails. The system is preventively replaced in order to prevent failure of unit 1 . The other unit referred to as unit 2 , is cheaper and easily replaceable. Unit 2 is only replaced when it fails. However, if its failure is within a threshold of the planned preventive system replacement, the system replacement is done in advance to avoid over replacements of unit 2 . A framework is proposed to jointly optimize the preventive replacement interval and the threshold for advanced replacement.
\end{abstract}

Keywords: preventive replacement; advanced replacement; failure replacement; threshold

(Submitted on October 1, 2017; Revised on November 7, 2017; Accepted on December 5, 2017)

(C) 2018 Totem Publisher, Inc. All rights reserved.

\section{Introduction}

In order to avoid the higher failure cost, a preventive replacement is often carried out in modern industries [1,2,4,5]. The agebased replacement policy has been widely used to calculate the optimal preventive replacement time [6,8]. However, most of these works considered systems consisting of a single unit [3]. In practice, a system may consist of a cheaper unit and a more expensive unit. The whole system is usually replaced in case the expensive unit fails, whereas the cheaper unit itself is only replaced if the cheaper unit fails [7].

In this paper, a system consisting of two units is considered. Unit 1 is more expensive and the system is replaced when it fails. Unit 1 is assumed to have increasing failure rate, and the system is preventive replaced in order to avoid too much failure cost. Unit 2 is cheaper, and unit 2 itself is only replaced when it fails. However, in order to avoid repetitive unit 2 replacement, it is assumed that the whole system preventive replacement can be done in advance if the failure of unit 2 is within a threshold of the planned preventive system replacement.

Section 2 presents the assumptions and notations. Section 3 models the system expected cost ratio in long term based on renewal theory. Section 4 presents a numerical example to solve the optimal replacement interval and advanced replacement threshold. Section 5 concludes.

\section{Assumptions and notations}

\subsection{Assumptions}

- The system consists of two independent units, in which the failure of unit 1 will lead to the system's replacement. Unit 2 itself is only replaced when it fails. Both the two units are subject to 0-1 logic failure mode.

- There are 3 types of replacement for the system: failure replacement, preventive replacement and advanced replacement. The replacement times are negligible.

- Failure replacement means that the system is replaced because of the failure of unit 1 .

* Corresponding author.

E-mail address: pengrui1988@ustb.edu.cn 
- If unit 1 has been working until preventive age $T$ without any failure, a preventive replacement is carried out for the system.

- If unit 2 is replaced at some time whose distance to preventive age $T$ is less than threshold $\tau$, then an advanced replacement for the whole system is taken.

- The failures for the two units are independent, and the unit failure can be detected immediately.

\subsection{Notations}

$\begin{array}{ll}T & \text { Age based replacement interval } \\ t_{f} & \text { Failure based replacement interval } \\ T_{A R} & \text { Advanced replacement interval } \\ \tau & \text { Threshold for advanced replacement } \\ X_{1} & \text { The duration of the life for unit } 1 \\ Y_{i}(i=1,2 \ldots m) & \text { The i-th duration for unit } 2 \\ T_{m}=\sum_{i=1} Y_{i} & \text { The total time during which the unit } 2 \text { has been replaced for } m \text { times } m=1,2 \ldots \\ f_{X}(x)^{=1} & \text { The pdf for } X \\ F_{X}(x) & \text { The cdf for } X \\ f_{y}\left(y_{i}\right) & \text { The pdf for } Y_{i}(i=1,2 \ldots) \\ F_{y}\left(y_{i}\right) & \text { The cdf for } Y_{i}(i=1,2 \ldots) \\ C_{I} & \text { Inspection cost } \\ C_{P} & \text { Preventive replacement cost } \\ C_{F 1} & \text { Failure replacement cost for unit } 1 \\ C_{F 2} & \text { Failure replacement cost for unit } 2 \\ m(m=0,1.2 \ldots) & \text { The total replacement times for the unit } 2 \text { strictly before the system is renewed } \\ P_{m}(t) & \text { The probability that the unit } 2 \text { has been replaced for } m \text { times at time } t\end{array}$

\section{The advanced replacement model}

\subsection{The general models}

Based on assumption2, the system will be replaced in 3 scenarios, as detailed below.

- Failure replacement

A failure of unit 1 occurs at $t_{f}$ before the preventive interval $T$ is reached. This scenario implies the following two exclusive possible cases:

(1) Unit 1 fails at $t_{f}\left(t_{f}<T_{A R}=T-\tau\right)$.The total time that unit 2 has been replaced for $m$ times before failure of unit 1 is represented by $T_{m}\left(T_{m}<t_{f}\right)$, as illustrated in Figure1(a);

(2) Unit 1 fails at $t_{f}\left(T_{A R}<t_{f}<T\right)$. The total time that unit 2 has been replaced for $m$ times before failure of unit 1 is represented by $T_{m}\left(T_{m}<T_{A R}\right)$, as illustrated in Figure1(b).

(a)

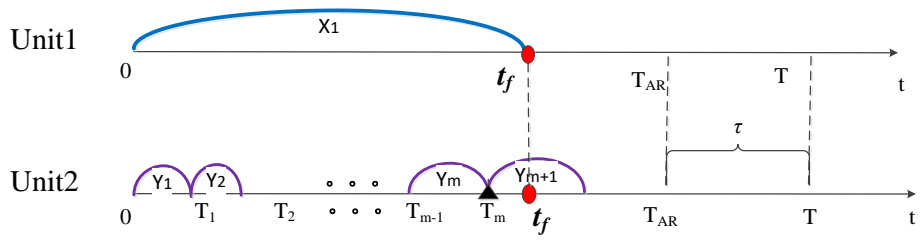

(b)

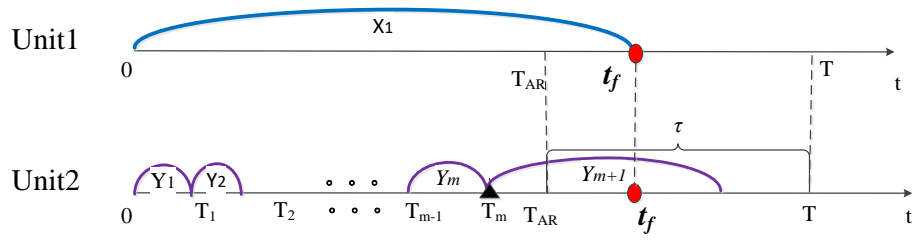

Figure 1. Renewal of the system due to a failure of unit $1, \bullet$ is the failure replacement time 
Thus, the corresponding probability is

$$
\begin{aligned}
& P_{1}(T, \tau)=P_{r}\left(t_{f}<T, t_{m}<\min \left(T-\tau, t_{f}\right), y_{m+1}>t_{f}-t_{m}\right) \\
& =\int_{0}^{T-\tau} \int_{0}^{t_{f}} f_{X_{1}}\left(t_{f}\right) f_{T_{m}}\left(t_{m}\right) R_{Y_{m+1}}\left(t_{f}-t_{m}\right) d t_{m} d t+\int_{T-\tau}^{T} \int_{0}^{T-\tau} f_{X_{1}}\left(t_{f}\right) f_{T_{m}}\left(t_{m}\right) R_{Y_{m+1}}\left(t_{f}-t_{m}\right) d t_{m} d t .
\end{aligned}
$$

The expected cost and length caused by a failure renewal are given by

$$
\begin{aligned}
& T C_{F R 1}(T, \tau)=\sum_{m=1}^{\infty}\left(\begin{array}{l}
\left(m C_{F_{2}}+C_{F_{1}}\right) \int_{0}^{T-\tau} \int_{0}^{t_{f}} f_{X_{1}}\left(t_{f}\right) f_{T_{m}}\left(t_{m}\right) R_{Y_{m+1}}\left(t_{f}-t_{m}\right) d t_{m} d t \\
+\left(m C_{F_{2}}+C_{F_{1}}\right) \int_{T-\tau}^{T} \int_{0}^{T-\tau} f_{X_{1}}\left(t_{f}\right) f_{T_{m}}\left(t_{m}\right) R_{Y_{m+1}}\left(t_{f}-t_{m}\right) d t_{m} d t
\end{array}\right) \\
& T L_{F R 1}(T, \tau)=\int_{0}^{T-\tau} \int_{0}^{t_{f}} t_{f} f_{X_{1}}\left(t_{f}\right) f_{T_{m}}\left(t_{m}\right) R_{Y_{m+1}}\left(t_{f}-t_{m}\right) d t_{m} d t+\int_{T-\tau}^{T} \int_{0}^{T-\tau} t_{f} f_{X_{1}}\left(t_{f}\right) f_{T_{m}}\left(t_{m}\right) R_{Y_{m+1}}\left(t_{f}-t_{m}\right) d t_{m} d t
\end{aligned}
$$

- Preventive replacement

Unit 1 is working without any failure until $T$. In this scenario, the latest replacement time $T_{m}$ for unit 2 before preventive replacement should not be within the advanced replacement threshold, as illustrated in Figure 2.

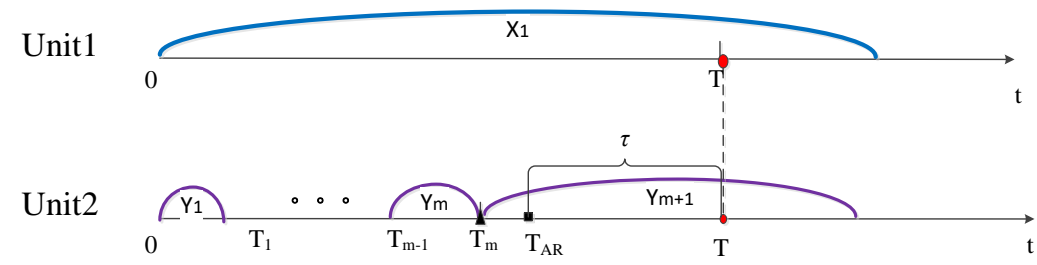

Figure 2. Renewal of the system due to preventive replacement, $\bullet$ is the preventive replacement time

Then, the probability of this scenario is

$$
\begin{aligned}
& P_{2}(T, \tau)=\operatorname{Pr}\left(X_{1}>T, t_{m}<T-\tau, y_{m+1}>T-t_{m}\right) \\
& =\int_{T}^{\infty} \int_{0}^{T-\tau} \int_{T-t_{m}}^{\infty} f_{X_{1}}(t) f_{T_{m}}\left(t_{m}\right) f_{Y_{m+1}}(y) d t d y d t_{m} \\
& =\int_{0}^{T-\tau} R_{X_{1}}(T) f_{T_{m}}\left(t_{m}\right) R_{Y_{m+1}}\left(T-t_{m}\right) d t_{m} .
\end{aligned}
$$

The expected cost and length caused by a preventive renewal is given by

$$
\begin{aligned}
& T C_{P R 1}(T, \tau)=\sum_{m=1}^{\infty}\left(\left(m C_{F_{2}}+C_{P R}\right) \int_{0}^{T-\tau} R_{X_{1}}(T) f_{T_{m}}\left(t_{m}\right) R_{Y_{m+1}}\left(T-t_{m}\right) d t_{m} d t\right), \\
& T L_{P R 1}(T, \tau)=\int_{0}^{T-\tau} T R_{X_{1}}(T) f_{T_{m}}\left(t_{m}\right) R_{Y_{m+1}}\left(T-t_{m}\right) d t_{m} d t
\end{aligned}
$$

- $\quad$ Advanced replacement

From assumption 5, an advanced replacement will be taken when the $m t h$ replacement for unit 2 is in the advanced replacement threshold while unit 1 is still in the working state, as illustrated by Figure 3.

Unit1

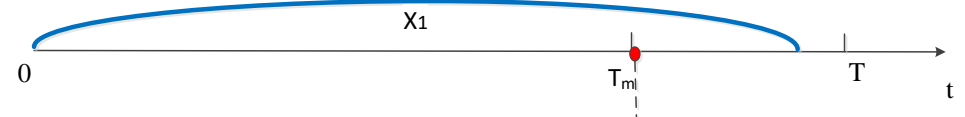

Unit2

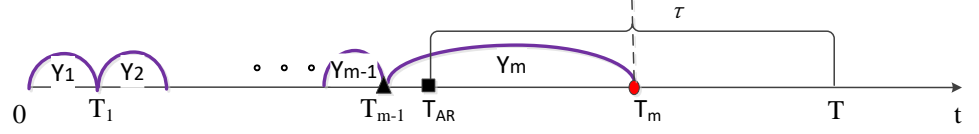

Figure 3. Renewal of the system due to advanced replacement, $\boldsymbol{\bullet}$ is the advanced replacement time 
For $m>1$, the probability of advanced replacement is

$$
\begin{aligned}
& P_{31}(T, \tau)=\operatorname{Pr}\left(X_{1}>t_{m}, T-\tau<t_{m}<T, 0<t_{m-1}<T-\tau\right) \\
& =\int_{0}^{T-\tau} \int_{T-\tau}^{T} \int_{t_{m}}^{\infty} f_{X_{1}}(t) f_{T_{m-1}}\left(t_{m-1}\right) f_{T_{m}}\left(t_{m} \mid t_{m-1}\right) d t d t_{m} d t_{m-1} \\
& =\int_{0}^{T-\tau} \int_{T-\tau}^{T} R_{X_{1}}\left(t_{m}\right) f_{T_{m-1}}\left(t_{m-1}\right) f_{T_{m}}\left(t_{m} \mid t_{m-1}\right) d t_{m} d t_{m-1} .
\end{aligned}
$$

Furthermore, the corresponding expected cost and length are

$$
\begin{aligned}
& T C_{A R 1}(T, \tau)=\sum_{m=2}^{\infty}\left(\left(m C_{F_{2}}+C_{P R}\right) \int_{0}^{T-\tau} \int_{T-\tau}^{T} R_{X_{1}}\left(t_{m}\right) f_{T_{m-1}}\left(t_{m-1}\right) f_{T_{m}}\left(t_{m} \mid t_{m-1}\right) d t_{m} d t_{m-1}\right) \\
& T L_{A R 1}(T, \tau)=\int_{0}^{T-\tau} \int_{T-\tau}^{T} t_{m} R_{X_{1}}\left(t_{m}\right) f_{T_{m-1}}\left(t_{m-1}\right) f_{T_{m}}\left(t_{m} \mid t_{m-1}\right) d t_{m} d t_{m-1}
\end{aligned}
$$

Specially, if $m=1$, it means that the time for the first replacement of unit 2 is within the threshold, and the probability for this case is

$$
\begin{aligned}
& P_{32}(T, \tau)=\operatorname{Pr}\left(X_{1}>t_{1}, T-\tau<t_{1}<T\right) \\
& =\int_{T-\tau}^{T} \int_{t_{1}}^{\infty} f_{X_{1}}(t) f_{T_{1}}\left(t_{1}\right) d t d t_{1}=\int_{T-\tau}^{T} R_{X_{1}}\left(t_{1}\right) f_{T_{1}}\left(t_{1}\right) d t_{1} .
\end{aligned}
$$

The expected cost and length for this case can be denoted by

$$
\begin{aligned}
& T C_{A R 1}(T, \tau)=\sum_{m=1}^{\infty}\left(\left(C_{F_{2}}+C_{P R}\right) \int_{T-\tau}^{T} R_{X_{1}}\left(t_{1}\right) f_{T_{1}}\left(t_{1}\right) d t_{1}\right), \\
& T L_{A R 1}(T, \tau)=\int_{T-\tau}^{T} t_{1} R_{X_{1}}\left(t_{1}\right) f_{T_{1}}\left(t_{1}\right) d t_{1} .
\end{aligned}
$$

\subsection{The expected cost per unit time}

With the corresponding probabilities derived in Section 3.1, the expected cost per unit time can be readily obtained

$$
\operatorname{ETC}(T, \tau)=\frac{T C_{F R 1}+T C_{P R 1}+T C_{A R 1}+T L_{A R 2}}{T L_{F R 1}+T L_{P R 1}+T L_{A R 1}+T L_{A R 2}}
$$

\section{A case study}

In this section, we provide a numerical example to illustrate the application of the presented approach. Consider a pump system that consists of a pump and a battery that is utilized to charge the pump. In this system, the pump is the primary unit with a relatively long lifetime. The failure of the pump means the failure of the whole system, while the battery itself is replaced when it fails. Also, the failure cost of the pump is far more than that of the battery. We assume the lifetime of the pump (unit 1) is subject to a weibull distribution $F_{X}(x)=1-\exp \left\{-\left(\frac{x}{\eta}\right)^{\beta}\right\}$, with an increasing failure rate with time and that of the battery (unit 2) is exponential distribution $f_{Y}(y)=\lambda e^{-\lambda y}$.

Since $Y_{i}(i=1,2 \ldots)$ is subject to exponential distribution, then $T_{m}=\sum_{i=1}^{m} Y_{i}$ can be described by Gamma distribution. When $m$ is an integer, a special case for Gamma distribution-Erlang distribution can represent the probability distribution function of $T_{m}$ as 


$$
f_{T_{m}}\left(t_{m}\right)=\left\{\begin{array}{lc}
\frac{1}{\lambda^{m} \Gamma(m)} x^{m-1} e^{-\frac{x}{\lambda}}, & \text { for } t_{m}>0, \quad m=1,2 \ldots \\
0, & \text { for } \quad t_{m}<0
\end{array}\right.
$$

The distribution parameters are shown in Table 1and the cost parameters are shown in Table 2.

Table 1. Distribution parameters
\begin{tabular}{|c|c|c|c|}
\hline parameters & $\beta$ & $\eta$ & $\lambda$ \\
\hline value & 1.5 & 100 & 0.2 \\
\hline \multicolumn{4}{|c|}{ Table 2. Cost parameters } \\
\hline parameters & $C_{f_{1}}$ & $C_{f_{2}}$ & $C_{P}$ \\
\hline value & 2000 & 50 & 150 \\
\hline
\end{tabular}

Figure 4 shows the variation of expected cost per unit time with preventive replacement interval $T$ and the advanced replacement threshold $\tau$.

For the given parametric setting, the optimal replacement interval $T$ and advanced replacement threshold are found to be $\left(T^{*}, \tau^{*}\right)=(29,5.22)$, indicating that the preventive replacement should be carried out at $T=29$ if no failure occurs before it and the advanced preventive replacement threshold $\tau=5.22$. The optimal expected long-run cost per unit time is $\operatorname{ETC}\left(\mathrm{T}^{*}, \tau^{*}\right)=24.3889$.

Then, we can make a comparison between the traditional maintenance policy without considering advanced preventive replacement. When $\tau=0$, the proposed policy degrades to traditional maintenance policy. Figure 5 shows the variation of expected cost per unit time only with preventive replacement interval $T$. The optimal replacement interval $T$ in this policy is $T^{*}=28$, and the related optimal expected long-run cost per unit time is $\operatorname{ETC}\left(\mathrm{T}^{*}\right)=24.3937$, which illustrates that our proposed method shows a better result. Note that though the difference for the ETC is small, the cost difference can be significant if there are many pump systems working for a long period. Say, a hospital may have thousands of pump systems, which typically work for decades.

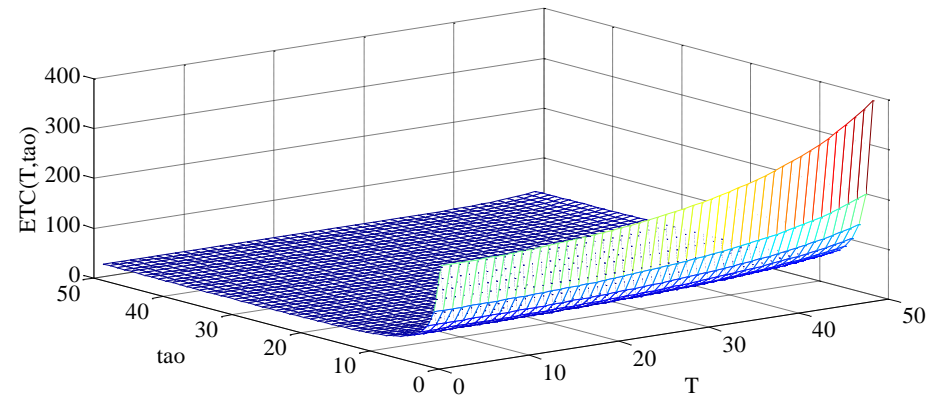

Figure 4. The expected cost per unit time varies with preventive replacement interval $T$ and the advanced replacement threshold $\tau$

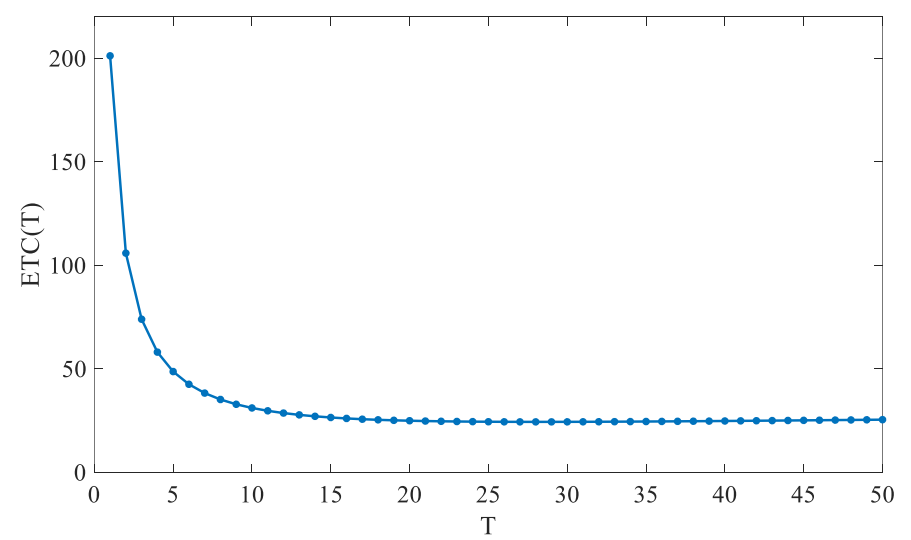

Figure 5. The expected cost per unit time varies with preventive replacement interval $T$ when $\tau=0$ 


\section{Conclusions}

This paper proposed an advanced replacement model for a two-unit system, where the failure of unit 1 requires the whole system to be replaced while unit 2 just needs to be replaced on its own once it fails. In order to prevent the sudden failure of unit 1, a preventive replacement interval was introduced. An advanced replacement policy was considered to avoid the over replacements of unit 2 . We analysed all the cases that can lead to the replacement of the system and derived the expected cost function per unit time. At last, an example was provided to illustrate the application of the presented approach. In the future, this work can be extended to cases where the units have degrading failures. In this case, joint optimization of the preventive maintenance interval, inspection interval, and advanced replacement threshold can be investigated.

\section{Acknowledgements}

The research reported here was partially supported by the NSFC under grant numbers 71671016, 71231001 and 71420107023 and by FRFCUs grant number FRF-GF-17-B14.

\section{References}

1. G. Levitin and A. Lisnianski, "Optimization of imperfect preventive maintenance for multi-state systems," Reliability Engineering \& System Safety, vol. 67, no. 2, pp. 193-203, February 2000.

2. X. Liu, W. Wang, F. Zhao and R. Peng, "Joint lot-size and preventive maintenance optimization for a production system," International Journal of Performability Engineering, vol. 11, no. 1, pp. 91-96, January 2015.

3. R. Peng, B. Liu, Q. Zhai and W. Wang, "Optimal maintenance strategy for system subject to two failure modes," Reliability Engineering \& System Safety, in press, doi: 10.1016/j.ress.2017.07.014.

4. L. Yang, X. Ma, R. Peng, Q. Zhai and Y. Zhao, "A preventive maintenance policy based on dependent two-stage deterioration and external shocks," Reliability Engineering \& System Safety, vol. 160, pp. 201-211, April2017.

5. R. Yang, F. Zhao, J. Kang, and X. Zhang, "An inspection optimization model based on a three-stage failure process," International Journal of Performability Engineering, vol. 10, no. 7, pp. 775-779, November 2014.

6. W. Wang, "A model to predict the residual life of rolling element bearings given monitored condition information to date," IMA Journal of Management Mathematics, vol. 13, no. 1, pp. 3-16, January 2002.

7. W. Wang, "Modelling condition monitoring inspection using the delay time concept," UK: University of Salford,1992.

8. W. Wang, F. Zhao and R. Peng, "A preventive maintenance model with a two-level inspection policy based on a three-stage failure process," Reliability Engineering \& System Safety, vol. 121, no. 1, pp. 207-220, January 2014.

Xiaoyang Ma is a Ph. D student in Donlinks School of Economics \& Management, University of Science \& Technology Beijing.

Xiaodong Zhang is a professor in Donlinks School of Economics \& Management, University of Science \& Technology Beijing.

Rui Peng is an associate professor in Donlinks School of Economics \& Management, University of Science \& Technology Beijing. He is an IEEE senior member, and in the editorial board of Reliability Engineering \& System Safety. 